\title{
Computers
} \& Structures

PERGAMON

\section{A finite point method for elasticity problems}

\author{
E. Oñate *, F. Perazzo ${ }^{1}$, J. Miquel \\ International Centre for Numerical Methods in Engineering, Universidad Politécnica de Cataluña, Gran Capitán s/n, 08034 Barcelona, \\ Spain
}

\begin{abstract}
The basis of the finite point method (FPM) for the fully meshless solution of elasticity problems in structural mechanics is described. A stabilization technique based on a finite calculus procedure is used to improve the quality of the numerical solution. The efficiency and accuracy of the stabilized FPM in the meshless analysis of simple linear elastic structural problems is shown in some examples of applications. (c) 2001 Elsevier Science Ltd. All rights reserved.
\end{abstract}

Keywords: Finite point method; Meshless method; Elasticity

\section{Introduction}

Mesh free techniques have become quite popular in computational mechanics. A family of mesh free methods is based on smooth particle hydrodynamic procedures $[1,2]$. These techniques, also called free lagrangian methods, are typically used for problems involving large motions of solids and moving free surfaces in fluids. A second class of mesh free methods derive from generalized finite difference (GFD) techniques [3,4]. Here the approximation around each point is typically defined in terms of Taylor series expansions and the discrete equations are found by using point collocation. Among a third class of mesh free techniques we find the so called diffuse element (DE) method [5], the element free Galerking (EFG) method [6,7], the reproducing kernel particle (RKP) method [8], the meshless local Petrov-Galerkin (MLPG) method $[9,10]$ and the method of finite spheres [11]. These methods use local interpolations for defining the approximate field around a point in terms of values in adjacent points, whereas the discretized system of equations is typically obtained by

\footnotetext{
${ }^{*}$ Corresponding author. Tel.: +34-93-205-7016; fax: +34-93401-6517.

E-mail address: onate@cimne.upc.es (E. Oñate). $U R L:$ http://www.cimne.upc.es.

${ }^{1}$ Professor at the Universidad Técnica Federico Santamaría, Valparaiso, Chile. Visiting Professor at CIMNE.
}

integrating the Galerkin variational form over a suitable background grid [5-10].

The finite point method (FPM) proposed in Refs. [12-16] is a truly meshless procedure. The approximation around each point is obtained by using standard moving least square techniques similarly as in DE and EFG methods. The discrete system of equations is obtained by sampling the governing differential equations at each point as in GFD methods.

The basis of the success of the FPM for solid and fluid mechanics applications is the stabilization of the discrete differential equations. The stable form found by the finite calculus (FIC) procedure presented in Refs. [18-23] corrects the errors introduced by the point collocation procedure, mainly next to boundary segments. In addition, it introduces the necessary stabilization for treating high convection effects and it also allows equal order velocity-pressure interpolations in fluid flow problems $[22,23]$. This paper extends preliminary successful work of the authors to derive a stabilized FPM for analysis of solid mechanics problems using the FIC approach [24].

The content of the paper is structured as follows. In Section 2 the basis of the FPM approximation is presented. The concept of the FIC procedure is detailed next. The discretization of the equilibrium equations in solid mechanics using a stabilized FPM via the FIC technique is described. The efficiency of the stabilized FPM is verified in several applications to the $2 \mathrm{D}$ and 3D analysis of simple linear elastic solids. 


\section{Interpolation in the finite point method}

Let $\Omega_{i}$ be the interpolation domain (cloud) of a function $u(x)$ and let $s_{j}$ with $j=1,2, \ldots, n$ be a collection of $n$ points with coordinates $x_{j} \in \Omega_{i}$. The unknown function $u$ may be approximated within $\Omega_{i}$ by

$u(x) \cong \hat{u}(x)=\sum_{l=1}^{m} p_{l}(x) \alpha_{l}=\mathbf{p}(x)^{\mathrm{T}} \boldsymbol{\alpha}$,

where $\boldsymbol{\alpha}=\left[\alpha_{1}, \alpha_{2}, \ldots \alpha_{m}\right]^{\mathrm{T}}$ and vector $\mathbf{p}(x)$ contains typically monomials, hereafter termed "base interpolating functions", in the space coordinates ensuring that the basis is complete. For a 2D problem we can specify

$\mathbf{p}=[1, x, y]^{\mathrm{T}} \quad$ for $m=3$

and

$\mathrm{p}=\left[1, x, y, x^{2}, x y, y^{2}\right]^{\mathrm{T}}$ for $m=6$ etc.

Function $u(x)$ can now be sampled at the $n$ points belonging to $\Omega_{i}$ giving
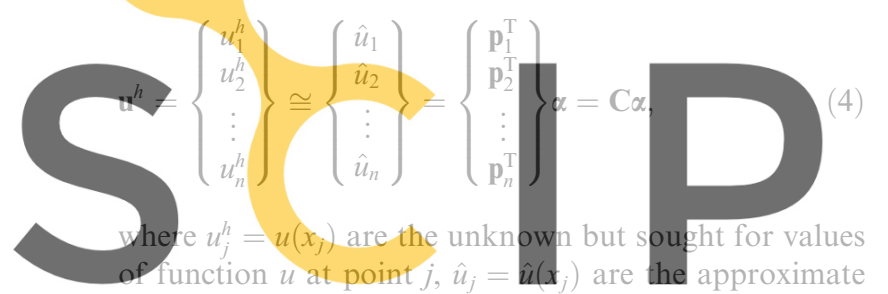

values, and $\mathrm{p}_{j}=\mathrm{p}\left(x_{j}\right)$.

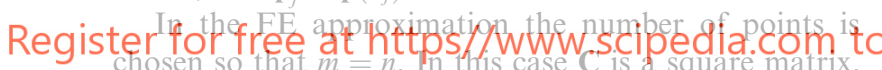

The procedure leads to the standard shape functions in the FEM [25].

If $n>m, \mathbf{C}$ is no longer a square matrix and the approximation cannot fit all the $u_{j}^{h}$ values. This problem can be simply overcome by determining the $\hat{u}$ values by minimizing the sum of the square distances of the error at each point weighted with a function $\varphi(x)$ as

$J=\sum_{j=1}^{n} \varphi\left(x_{j}\right)\left(u_{j}^{h}-\hat{u}\left(x_{j}\right)\right)^{2}=\sum_{j=1}^{n} \varphi\left(x_{j}\right)\left(u_{j}^{h}-\mathbf{p}_{j}^{\mathrm{T}} \boldsymbol{\alpha}\right)^{2}$,

with respect to the $\alpha$ parameters. This approximation is termed weighted least square (WLS) interpolation. Note that for $\varphi(x)=1$ the standard least square (LSQ) method is reproduced.

Function $\varphi(x)$ is usually built in such a way that it takes a unit value in the vicinity of the point $i$ typically called "star node" where the function (or its derivatives) are to be computed and vanishes outside a region $\Omega_{i}$ surrounding the point (Fig. 1). The region $\Omega_{i}$ can be used to define the number of sampling points $n$ in the interpolation region. In all numerical examples presented in this paper, the normalized Gaussian weight function $\varphi(x)$ is used. Of course $n \geqslant m$ is always required

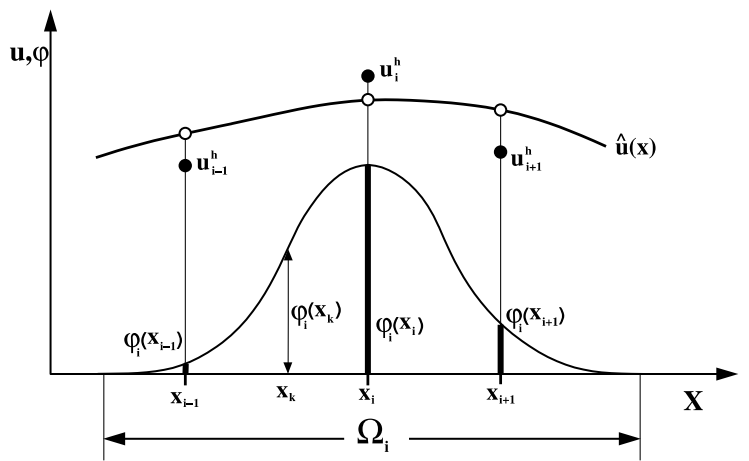

Fig. 1. WLS procedure.

in the sampling region and if equality occurs no effect of weighting is present and the interpolation is the same as in the LSQ scheme. A discussion on different possibilities for selecting the weighting function $\varphi(x)$ can be found in Refs. $[12,13,17]$.

Standard minimization of Eq. (5) with respect to $\alpha$ gives
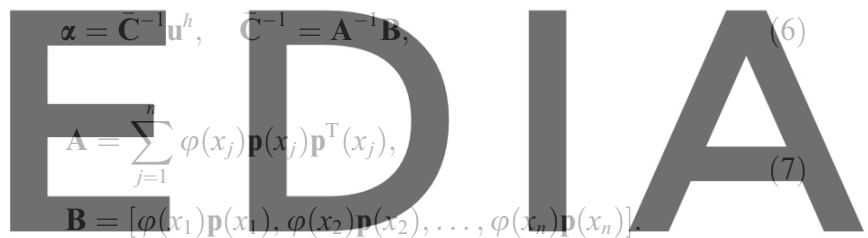
down final approximation is obtained by substituting $\alpha$

$$
\hat{u}(x)=\mathbf{p}^{\mathrm{T}} \overline{\mathbf{C}}^{-1} \mathbf{u}^{h}=\mathbf{N}^{\mathrm{T}} \mathbf{u}^{h}=\sum_{j=1}^{n} N_{j}^{i} u_{j}^{h},
$$

where the "shape functions" are

$N_{j}^{i}(x)=\sum_{l=1}^{m} p_{l}(x) \bar{C}_{l j}^{-1}=\mathbf{p}^{\mathrm{T}}(x) \overline{\mathbf{C}}^{-1}$.

It must be noted that accordingly to the least square character of the approximation

$u\left(x_{j}\right) \simeq \hat{u}\left(x_{j}\right) \neq u_{j}^{h}$,

i.e. the local values of the approximating function do not fit the nodal unknown values (Fig. 1). Indeed $\hat{u}$ is the true approximation for which we shall seek the satisfaction of the differential equation and the boundary conditions and $u_{j}^{h}$ are simply the unknown parameters sought.

The WLS approximation described above depends on a great extend on the shape and the way to apply the weighting function. The simplest way is to define a fixed function $\varphi(x)$ for each of the $\Omega_{i}$ interpolation domains $[13,14,17]$. 
Let $\varphi_{i}(x)$ be a weighting functions satisfying

$$
\begin{array}{ll}
\varphi_{i}\left(x_{i}\right)=1, & \\
\varphi_{i}(x) \neq 0 & x \in \Omega_{i}, \\
\varphi_{i}(x)=0 & x \notin \Omega_{i} .
\end{array}
$$

Then the minimization of the square distance becomes

$J_{i}=\sum_{j=1}^{n} \varphi_{i}\left(x_{j}\right)\left(u_{j}^{h}-\hat{u}\left(x_{j}\right)\right)^{2} \quad$ minimum.

The expression of matrices $\mathbf{A}$ and $\mathbf{B}$ coincide with Eq. (7) with $\varphi\left(x_{j}\right)=\varphi_{i}\left(x_{j}\right)$.

Note that according to Eq. (1), the approximate function $\hat{u}(x)$ is defined in each interpolation domain $\Omega_{i}$. In fact, different interpolation domains can yield different shape functions $N_{i}^{i}$. As a consequence a point belonging to two or more overlapping interpolation domains has different values of the shape functions which means that $N_{j}^{i} \neq N_{i}^{k}$. The interpolation is now multivalued within $\Omega_{i}$ and, therefore for any useful approximation a decision must be taken limiting the choice to a single value. Indeed, the approximate function $\hat{u}(x)$ will be typically used to provide the value of the unknown function $u(x)$ and its derivatives in only specif interpolation domain. For ins location we may limit the validit a) single point $x_{i}$. It is precisely found this meshless m

The definition of the points within a cloud for 2D

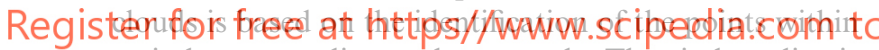
a circle surrounding each star node. The circle radius is defined so that a minimum number of points is located within the circle domain $(n>3$ and $n>6$ for linear and quadratic clouds, respectively, etc.). Obviously, the circle becomes a sphere for $3 \mathrm{D}$ clouds.

\section{Discretization of governing equations}

Let us assume a problem governed by the following set of differential equations

$A\left(u_{j}\right)=0 \quad$ in $\Omega$

with boundary conditions

$u_{j}-\bar{u}_{j}=0 \quad$ on $\Gamma_{u}$,

$B\left(u_{j}\right)=0 \quad$ on $\Gamma_{t}$.

In above $A$ is a differential operator defining the governing differential equations to be satisfied on the domain $\Omega$ with boundary $\Gamma=\Gamma_{t} \cup \Gamma_{\phi}, B$ is the differential operator defining the boundary conditions at the Neumann boundary $\Gamma_{t}, u_{j}$ are the unknown variables with prescribed values $\bar{u}_{j}$ at the Dirichlet boundary $\Gamma_{u}$, $j=1,2, \ldots, N_{\mathrm{v}}$, where $N_{\mathrm{v}}$ is the number of variables. In solid mechanics applications $u_{j}$ are the displacements and $A$ and $B$ are the equilibrium equations to be satisfied in the domain $\Omega$ and the boundary $\Gamma_{t}$ where tractions are prescribed, respectively.

The discretized system of equations in the FPM is found by substituting the approximation (8) into Eqs. (13a)-(13c) and collocating the differential equation at each point in the analysis domain. This gives

$$
\begin{aligned}
& {\left[A\left(\hat{u}_{j}\right)\right]_{p}=0 \quad p=1,2 \ldots N_{r},} \\
& {\left[\hat{u}_{j}\right]_{s}-\bar{u}_{j}=0 \quad s=1,2 \cdots N_{u},} \\
& {\left[B\left(\hat{u}_{j}\right)\right]_{r}=0 \quad r=1,2 \ldots N_{t} .}
\end{aligned}
$$

In above $N_{u}$ and $N_{t}$ are the number of points located on the boundaries $\Gamma_{u}$ and $\Gamma_{t}$, respectively and $N_{r}$ is rest of the points in $\Omega$ not belonging to any of the boundaries $\Gamma_{u}$ and $\Gamma_{t}$.

Eq. (14) lead to a system of algebraic equations of the form

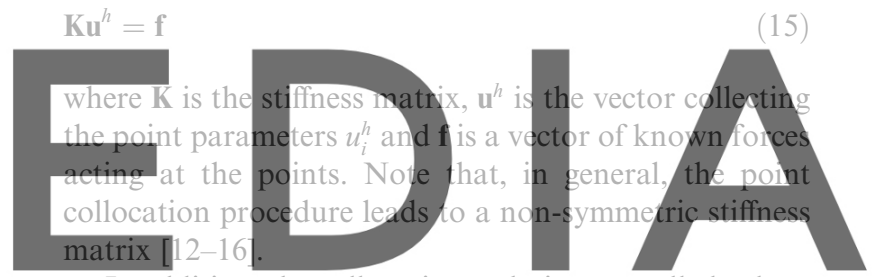

In addition, the collocation technique usually leads to an ill-conditioned system of equations and the solution

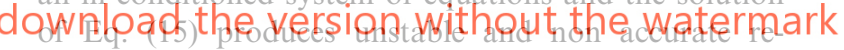
sults. This is mainly due to the incapacity of the point collocation method to satisfy precisely the equilibrium equations over a cloud of points of finite size, just by sampling the equations at the star node in the cloud. Indeed, these deficiencies are more pronounced in clouds next to a boundary segment due to the usual lack of symmetry of the clouds in boundary regions.

The deficiencies of the point collocation procedure can be overcome by using some kind of stabilization procedure. This requires the modification of the system of equations (15) in a clever form to avoid the ill-conditioning mentioned above. Most stabilization methods derived for collocation schemes are based on adding to the original equations new terms which are residualbased, i.e. terms which are a function of the governing equations themselves, thus ensuring consistency of the approach. The selection of these terms is somehow heuristic and there is lack of a general stabilization procedure [26].

In our work we have used a stabilized form of the governing equations derived from the FIC procedure described in Refs. [18-24]. The FIC method is based on imposing the typical balance laws of mechanics over a domain of finite size. The unknown fields are then 
approximated within the finite domain using a Taylor series expansion, retaining higher order terms than those used in the standard infinitesimal approach. This introduces naturally new terms in the governing differential equations which have stabilization features. The stabilized form of Eqs. (13a)-(13c) using the FIC method reads [18]

$A-\frac{1}{2} h_{k} \frac{\partial A}{\partial x_{k}}=0$ in $\Omega$,

$u_{j}-\bar{u}_{j}=0 \quad$ on $\Gamma_{u}$,

$B-\frac{1}{2} h_{k} n_{k} A=0 \quad$ on $\Gamma_{t}$,

where $n_{k}$ are the components of the unit normal to the boundary $\Gamma_{t}$ and $h_{k}$ are the dimensions of the balance domain (also called characteristic length parameters) with $k=1,2,3$ for $3 \mathrm{D}$ problems. The underlined terms in Eqs. (16a) and (16c) introduce the necessary stabilization in the governing equations at discrete level. It is interesting to note that Eqs. (16a)-(16c) are the starying point for deriving methods (FEMs) for advectionnamic problems. Here the new troduced by the FIC technique also instabilities due to convection sibility requirement $[18,22,2 \xi]$. The stabilized equations (16a)-(16c) have also been found useful for

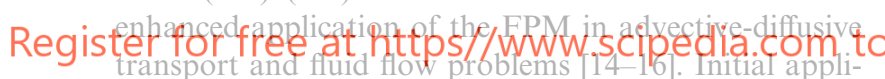
cations of the FIC method for the solution of solid mechanics problems using the FPM were reported in Ref. [24]. The underlying ideas of the FIC method have been recently used by Bonet and Kulasegaram to derive stabilized point integrated meshless methods for elliptic equations using SPH techniques [27]. The efficiency of the FIC stabilization procedure for the application of the FPM in elasticity problems is shown in this paper.

The discretized system of stabilized equations in the FPM is found by substituting the approximation (8) into Eqs. (16a)-(16c) and collocating the differential equations at each point in the analysis domain. This gives

$$
\begin{aligned}
& {\left[A\left(\hat{u}_{j}\right)-\frac{1}{2} h_{k} \frac{\partial}{\partial x_{k}} A\left(\hat{u}_{j}\right)\right]_{p}=0 \quad p=1,2 \ldots N_{r},} \\
& {\left[\hat{u}_{j}\right]_{s}-\bar{u}_{j}=0 \quad s=1,2 \ldots N_{u},} \\
& {\left[B\left(\hat{u}_{j}\right)-\frac{1}{2} h_{k} n_{k} A\left(\hat{u}_{j}\right)\right]_{r}=0 \quad r=1,2 \ldots N_{t} .}
\end{aligned}
$$

The discretized system of equations (17) can be written in the standard matrix form

$$
\left(\mathbf{K}+\mathbf{K}_{s}\left(h_{k}\right)\right) \mathbf{u}^{h}=\mathbf{f},
$$

from where the values of the nodal parameters $u_{i}^{h}$ can be found.

Matrix $\mathbf{K}$ in Eq. (18) denotes the stiffness matrix of Eq. (15) excluding the stabilization terms. The effect of these terms is accounted for in the new stabilization stiffness matrix $\mathbf{K}_{s}$ which is a function of the characteristic length parameters.

The Dirichlet boundary conditions (16b) are introduced in the solution of Eq. (18) simply by prescribing the values of the nodal parameters to the fixed displacement values. Indeed this is an approximation as the WLS interpolation does not match the nodal displacement values (Fig. 1). A more accurate although expensive procedure can be based in modifying the WLS approximation at the boundary clouds so that the displacement values at the boundary points satisfy exactly the Dirichlet condition. Further details on the implementation of the Dirichlet boundary conditions can be found in Refs. [14,15].

The computation of the characteristic length parameters is a critical issue in order to increase the accuracy of the stabilized solution. An optimum choice of the characteristic length values following a diminishing

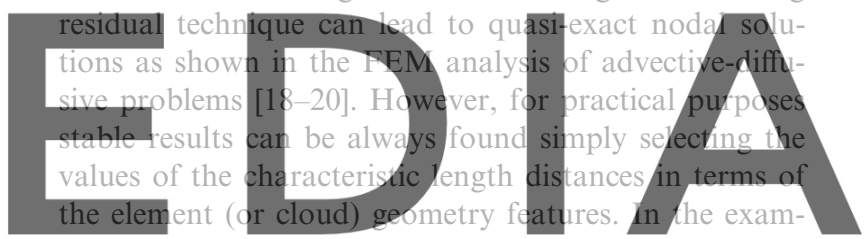
ples shown in the paper, the value $h_{i}=d_{i}^{\max }$ has been chosen where $d_{i}^{\max }$ is the largest distance along the $i$ th dowdrate the versipntawitheuit theidwatermark neighbours (Fig. 2).

\section{Stabilized finite point method for elasticity problems}

Following the ideas presented in the previous section the stabilized equations for elasticity problems can be written as

$\frac{\partial \sigma_{i j}}{\partial x_{j}}+b_{i}-\frac{1}{2} h_{k} \frac{\partial}{\partial x_{k}}\left(\frac{\partial \sigma_{i j}}{\partial x_{j}}+b_{i}\right)=0 \quad$ in $\Omega$,

$u_{j}-\bar{u}_{j}=0 \quad$ in $\Gamma_{u}$,

$\sigma_{i j} n_{j}+t_{i}-\frac{1}{2} h_{k} n_{k}\left(\frac{\partial \sigma_{i j}}{\partial x_{j}}+b_{i}\right)=0 \quad$ in $\Gamma_{t}$,

with $i, j, k=1,2,3$ for 3D problems. The precise derivation of Eqs. (19a)-(19c) can be found in Ref. [18].

In above, $u_{i}$ are the displacements along the cartesian coordinate directions, $\bar{u}_{j}$ are prescribed displacement values over the Dirichlet boundary $\Gamma_{u}, b_{i}$ and $t_{i}$ are prescribed body forces and tractions over the domain $\Omega$ 

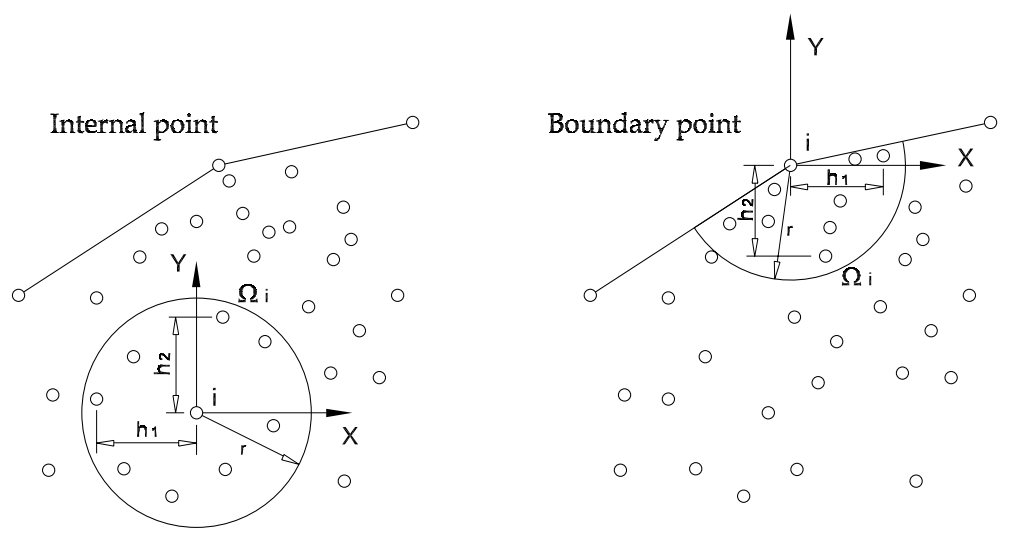

Fig. 2. Point search procedure for 2D quadratic clouds and definition of characteristic length distances for interior and boundary points.

and the Neumann boundary $\Gamma_{t}$, respectively and $\sigma_{i j}$ are the stresses which are related to the displacements by the standard Hook's law. In matrix form

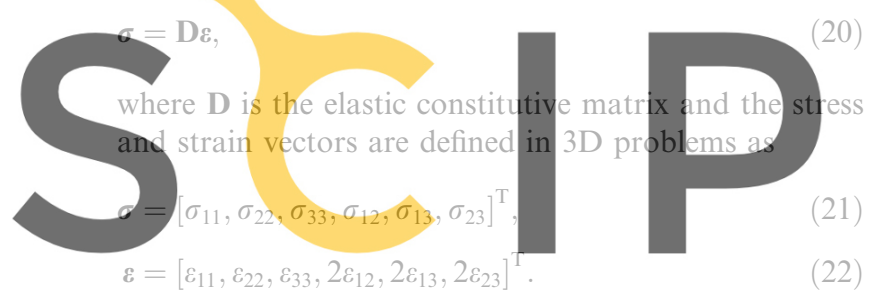

Register for free at https//www.scipedia.com to
The strains $\varepsilon_{i j}$ are relatted to the displacements by

$\varepsilon_{i j}=\frac{1}{2}\left(\frac{\partial u_{i}}{\partial x_{j}}+\frac{\partial u_{j}}{\partial x_{i}}\right)$

As earlier mentioned $h_{k}$ are the characteristic length parameters affecting the stabilization terms in Eqs. (19a) and (19c). As usual $n_{k}$ are the components of the unit normal to the boundary.

Note that the stabilization terms require the computation of the third and second derivatives of the displacements in Eqs. (19a) and (19c), respectively. This must be taken into account when choosing the order of the interpolation for the displacements.

In our work we have tested the efficiency of the stabilized FPM using quadratic interpolations for the displacement field (i.e. stabilization term in Eq. (19a) are zero). The same good results were obtained with the cubic interpolation neglecting the stabilization terms in Eq. (19a). This supports the fact that the key stabilization terms are those emanating from the Neumann boundary conditions (Eq. (19c)) whereas the stabilization terms in the equilibrium equation on the domain $\Omega$ can be neglected for practical purposes. This is also consistent with the nature of the ill-conditioning of the equations due mainly to the lack of symmetry of the points in clouds next to boundary segments.

Examples of the performance of the stabilized FPM in a number of 2D and 3D elasticity problems using

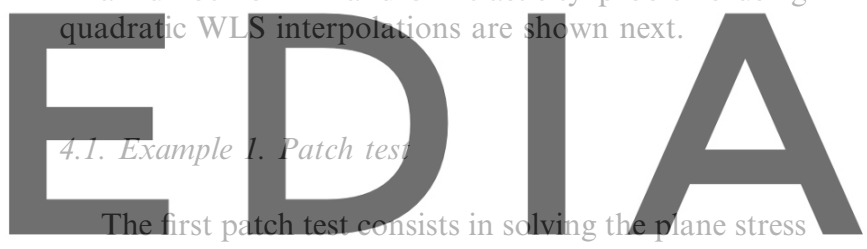

equations on a square domain of $2 \times 2$ units discretized

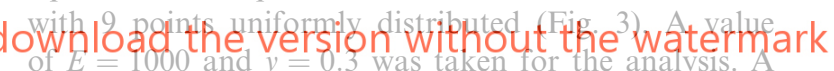
prescribed displacement $u=v=x+y$ is assigned to the eight boundary nodes. A quadratic displacement

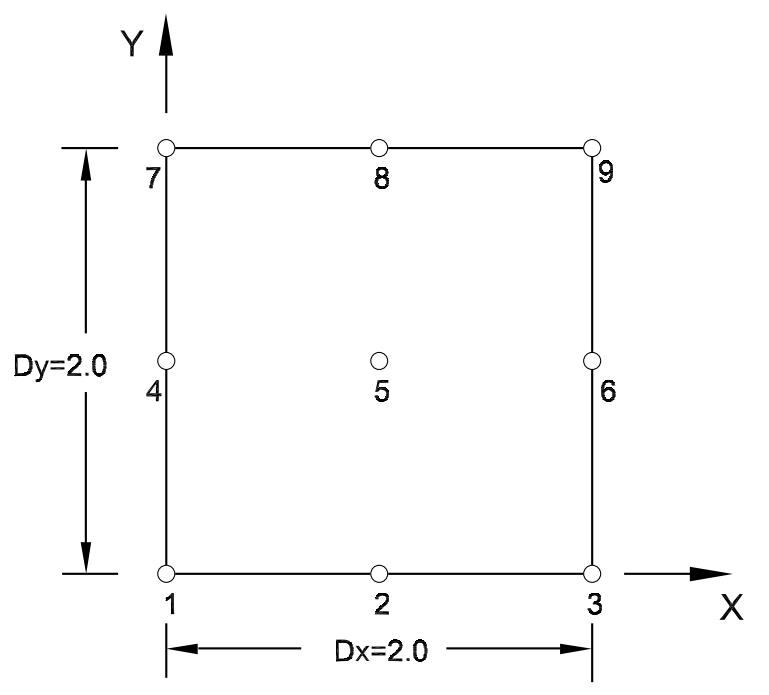

Fig. 3. Patch test with regular grid of 9 points. 
Table 1

Coordinates of point 5 for the patch test with regular grid of 9 points

Coordinates of point 5

$(1.0,1.0)$

$(1.2,0.35)$

$(0.22,0.15)$

$(1.87,1.9)$

Exact values are obtained for the displacements and the stresses at node 5

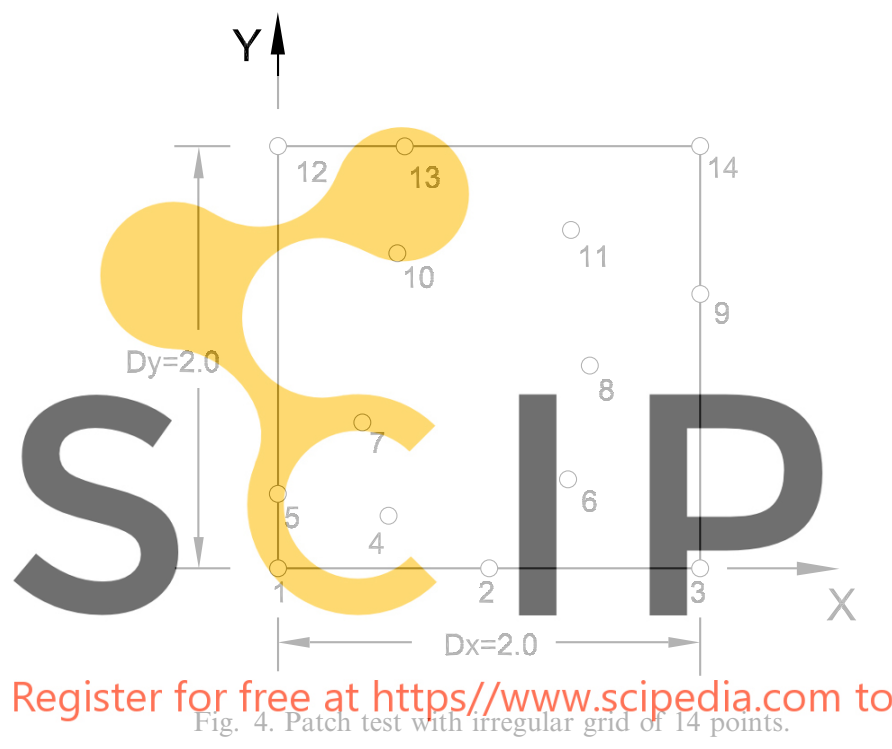

interpolation $(m=6)$ was chosen for the analysis. The displacements and the stresses computed at the central node coincide precisely with the exact solution. Identical accuracy was obtained by changing the coordinates of the central node as shown in Table 1 .

The same problem was solved using an irregular grid of 14 points distributed as shown in Fig. 4. Again, the exact analytical values for the linear displacement field and the constant stress field are obtained at all the internal nodes (see Table 2).

\subsection{Example 2. Rectangular domain under prescribed boundary tractions}

Fig. 5 shows the geometry of the domain, the boundary conditions, the material properties and the discretization into a uniform grid of 28 points. The plane stress assumption was chosen with a unit thickness and values of $E=1$ and $v=0.25$. The problem was solved for the cases of uniform and linear tractions at the boundary for $x=6$. The results for the nodal displacements and stresses using the stabilized FPM with quadratic clouds coincide with the analytical values for both loading cases. The horizontal displacement contours for the uniform traction case and the $\sigma_{x}$ stress contours for the linear traction case obtained with the stabilized FPM are shown in Fig. 6.

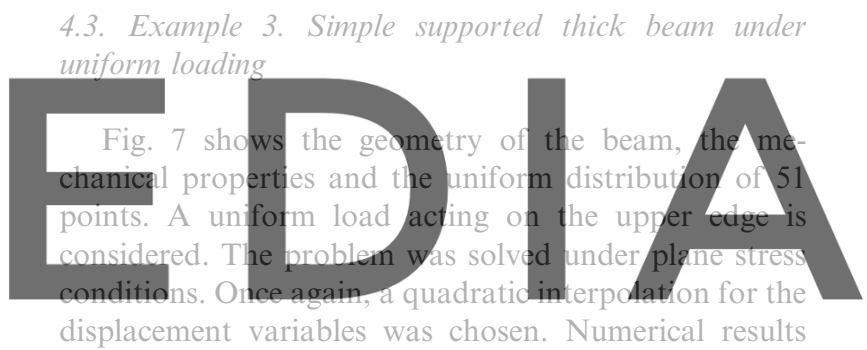

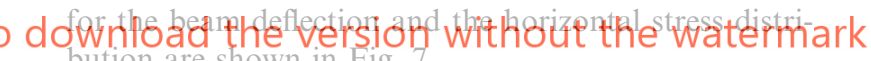 button are shown in $\mathrm{F}$ ig.}

The same problem was analyzed with the FEM using a structured mesh of 68 linear piane stress triangles (CST element) [25] based on the same point distribution. Nodal stresses have been obtained by standard nodal averaging of element values. Comparison of the errors for the central deflection and the maximum $\sigma_{x}$ stress gives some advantage to the stabilized FPM results (see Table 3).

The convergence of the maximum horizontal stress and the maximum deflection value with the number of

Table 2

Coordinates and numerical results for a patch test with an irregular grid of 14 points $^{\mathrm{a}}$

\begin{tabular}{llllll}
\hline Points & Coordinates & Displacements $(u=v)$ & $\sigma_{x}\left(\times 10^{4}\right)$ & $\sigma_{y}\left(\times 10^{4}\right)$ & $\tau_{x y}\left(\times 10^{3}\right)$ \\
\hline 4 & $(0.5,0.25)$ & 0.75 & 0.142857 & 0.142857 & 0.76923 \\
6 & $(1.4,0.45)$ & 1.85 & 0.142857 & 0.142857 & 0.76923 \\
7 & $(0.38,0.7)$ & 1.08 & 0.142857 & 0.142857 & 0.76923 \\
8 & $(1.5,1.0)$ & 2.5 & 0.142857 & 0.142857 & 0.76923 \\
10 & $(0.55,1.5)$ & 2.05 & 0.142857 & 0.142857 & 0.76923 \\
11 & $(1.38,1.6)$ & 2.98 & 0.142857 & 0.142857 & 0.76923 \\
\hline
\end{tabular}

${ }^{a}$ All numerical results coincide with the analytical values. 
(a)

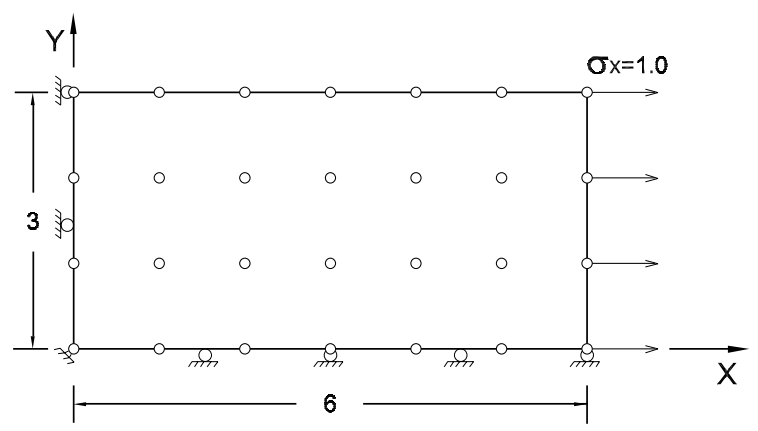

(b)

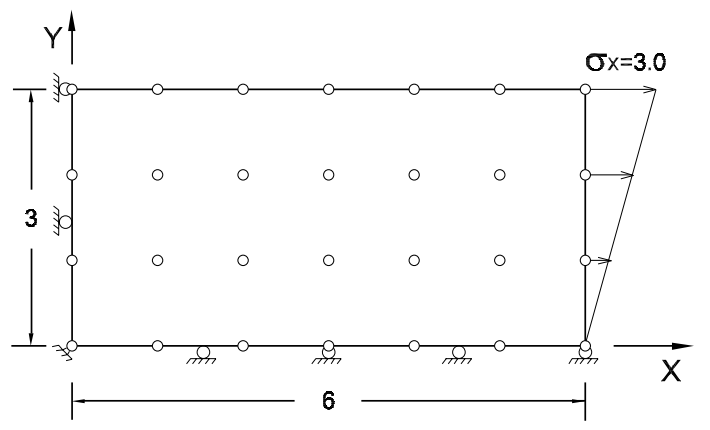

Fig. 5. Points arrangement of rectangular domain under prescribed boundary tractions. (a) Uniform tractions; (b) linear tractions.

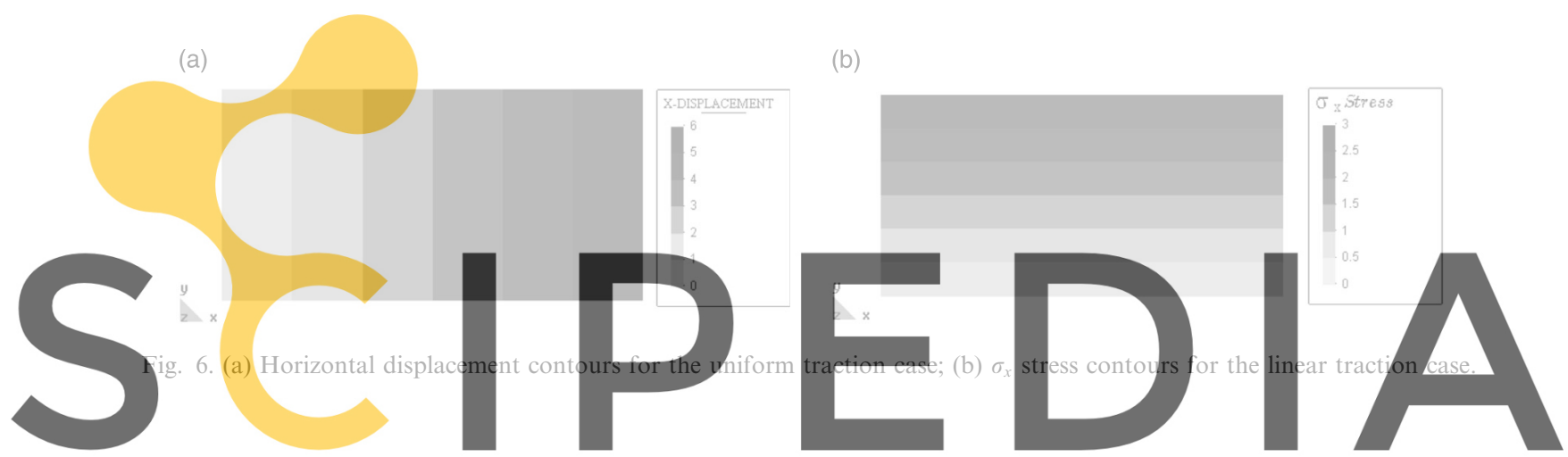

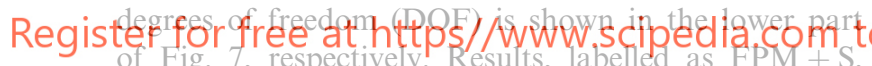
correspond to those obtained with the stabilized FPM described in the paper, whereas those listed as FPMI were obtained neglecting the stabilization terms (i.e. the terms involving the characteristic length parameters in Eq. (17)). Note the beneficial effect of the stabilization terms leading to results which are more accurate than those obtained by the standard FEM in this case.

\subsection{Example 4. Square domain with circular hole under tension}

Fig. 8 shows the geometry of the domain and the loading. One quarter of the domain is analyzed only due to symmetry. Plane strain conditions were assumed for the analysis. The problem has been solved with the quadratic FPM using two unstructured grids of 36 and 60 points. Contours of the horizontal stress obtained with the stabilized FPM are shown for the two grids. Results for the maximum horizontal stress at the upper tip of the hole obtained with the stabilized FPM compare well with the analytical value of $\sigma_{x}=3.0$ [28]. The FPM results also compare very favourably with those

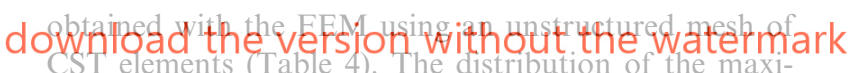
mum horizontal stress along the line $x=0$ is also shown in Fig. 8 for the two grids studied. Note the greater accuracy of the stabilized FPM solution versus the standard FPM and FEM solutions.

The distribution of the horizontal stress along the line $x=0$ for the two unstructured grids studied is shown in Fig. 9.

\subsection{Example 5. Thick cantilever under end point load}

Fig. 10 shows the geometry of the cantilever beam and the material properties. The end load of $P=1.0$ was applied as a parabolic tangential stress acting at the boundary for $x=8.0$. Plane stress conditions were assumed.

The problem was solved with regular and irregular grids of 55, 165 and 333 points using quadratic clouds. The distribution of points for the regular and irregular grids of 55 points are shown in Fig. 10. The contours of the vertical displacement and the $\sigma_{x}$ stress obtained with the regular grid of 333 points are plotted in Fig. 11. Very similar results were obtained using an irregular grid. 
(a)

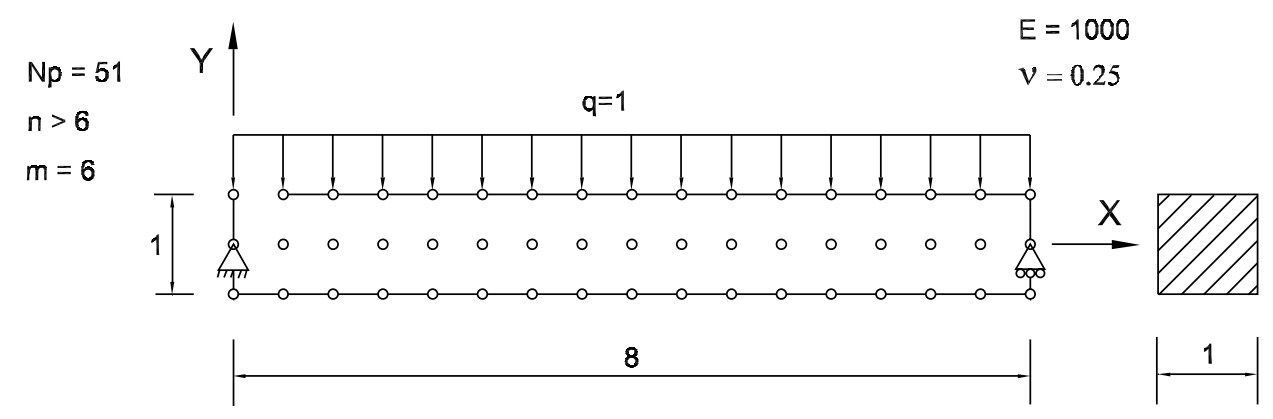

(b)

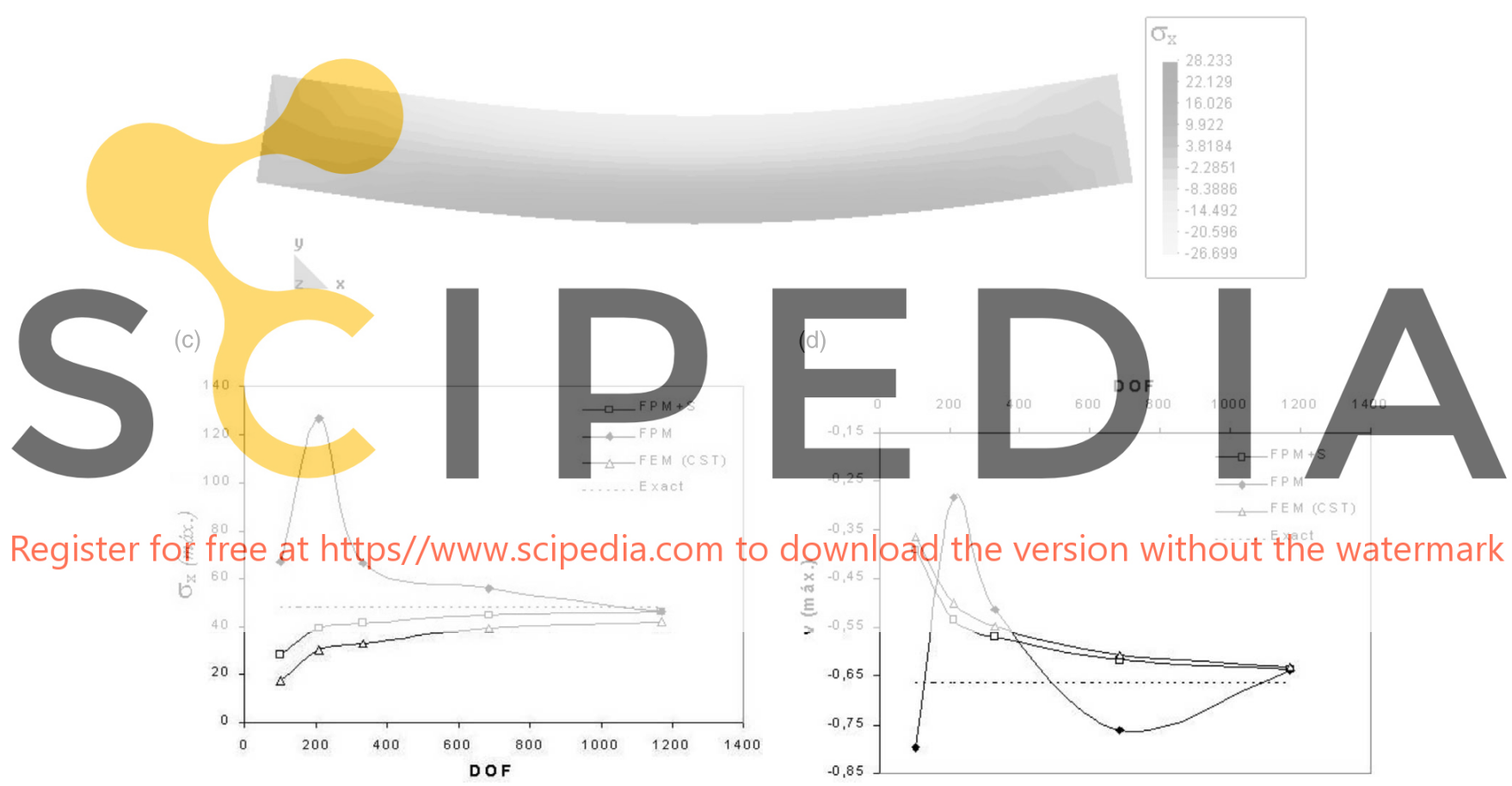

Fig. 7. Simple supported beam analyzed with FPM and CST finite elements. (a) Beam geometry, loading and regular grid of 51 points; (b) horizontal stress contours; (c) convergence of the maximum horizontal stress and (d) convergence of the central deflection with the number of DOF. Exact solution refers to classical beam theory [28].

Table 3

Simple supported beam: numerical results for grid of 51 points (68 CST finite elements)

\begin{tabular}{lllll}
\hline \multicolumn{2}{l}{ Central deflection error } & & \multicolumn{2}{l}{ Error in maximum $\sigma_{x}$ stress } \\
\cline { 1 - 3 } \cline { 5 - 6 } FPM $+\mathrm{S}$ & FEM & & FPM $+\mathrm{S}$ & FEM \\
\hline $19 \%$ & $21 \%$ & & $19 \%$ & $38 \%$ \\
\hline
\end{tabular}

Fig. 12 shows a plot of the shear stress distribution at the beam center obtained with the different regular grids.
The convergence of the displacement and the $\sigma_{x}$ stress using regular and irregular grids is plotted in Figs. 13 and 14 . The error was measured by the quadratic norm of the differences between point values and the exact solution. Distance $h$ in Figs. 13 and 14 denotes to the average radial distance for all the clouds in a grid. The slope of the convergence curves for the displacements $u$ and $v$ are found to be uniform and $\simeq 2$ for regular grids and $\simeq 3$ for irregular grids. It is remarkable that similar convergence rates are obtained for the $\sigma_{x}$ stress for both regular and irregular grids. 
(a)
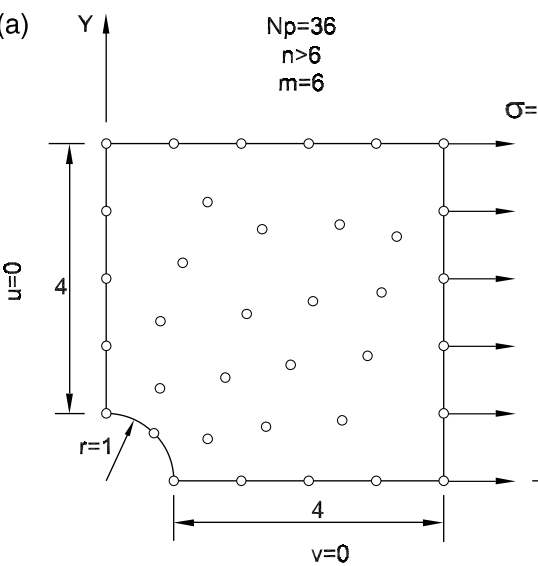

$\sigma=1$

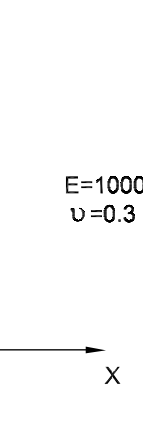

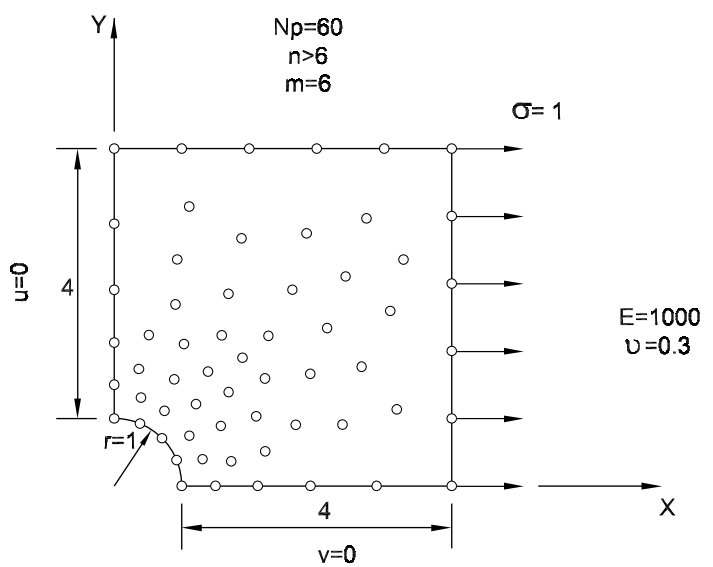
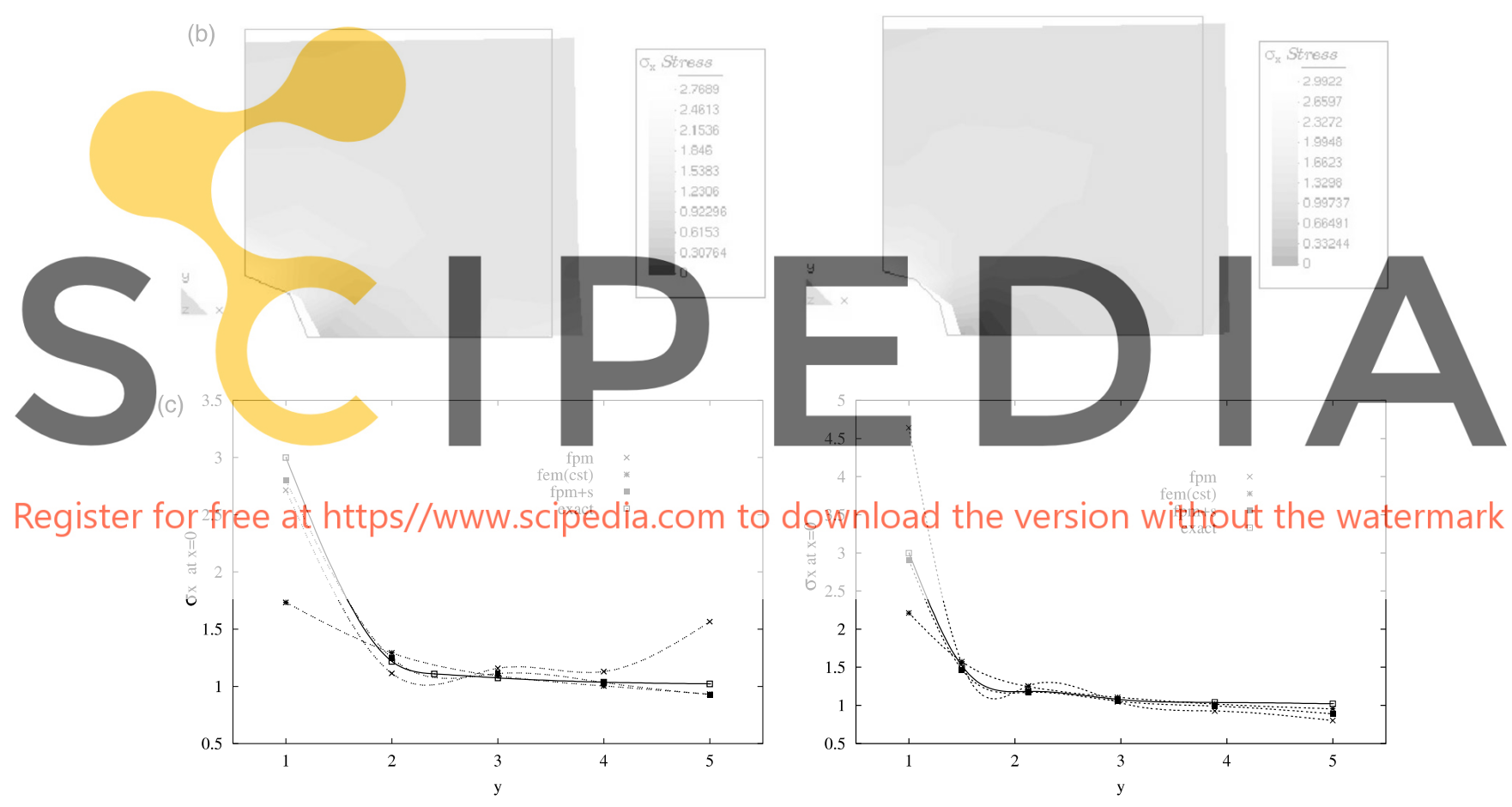

download the version without the watermark

Fig. 8. Square plate with a circular hole under tension analyzed with FPM and FEM (CST elements) $(E=1000, v=0.3)$. (a) Plate geometry and loading. Unstructured grids of 36 and 60 points; (b) $\sigma_{x}$ stress contours displayed over the deformed shapes obtained for the two grids studied; (c) distribution of maximum horizontal stress along the line $x=0$ for the two unstructured grids of 36 and 60 points.

Table 4

Square plate with circular hole: error in the maximum horizontal stress obtained with the stabilized EPM $($ FPM + S) and the FEM

\begin{tabular}{lllll}
\hline $\begin{array}{l}36 \\
\text { triangles }\end{array}$ & points $-50 \mathrm{CST}$ & & \multicolumn{2}{l}{$\begin{array}{l}60 \text { points }-94 \\
\text { triangles }\end{array}$} \\
\cline { 1 - 2 } \cline { 5 - 6 } FPM $+\mathrm{S}$ & FEM & & FPM $+\mathrm{S}$ & FEM \\
\hline $6 \%$ & $38 \%$ & & $1.5 \%$ & $21 \%$ \\
\hline
\end{tabular}

4.6. Example 6. 3D prismatic solid under end bending moments

Fig. 15 shows the geometry of the prismatic solid and the material properties. The end bending moments were modelled by an equivalent linear traction acting at the ends. An eight of the solid was analyzed only due to symmetry. A regular grid of $6 \times 5 \times 4$ points was chosen as shown in Fig. 15. Fig. 16 shows contours of the displacement along the longitudinal $z$-axis and the $\sigma_{z}$ stress. 


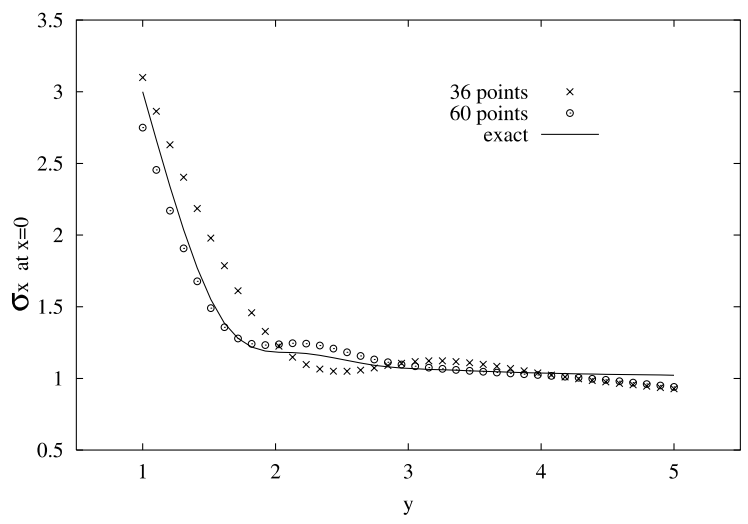

Fig. 9. Square plate with circular hole. Distribution of the horizontal stress along the line $x=0$ for the grids of 36 and 60 points. The exact distribution is also plotted [28].

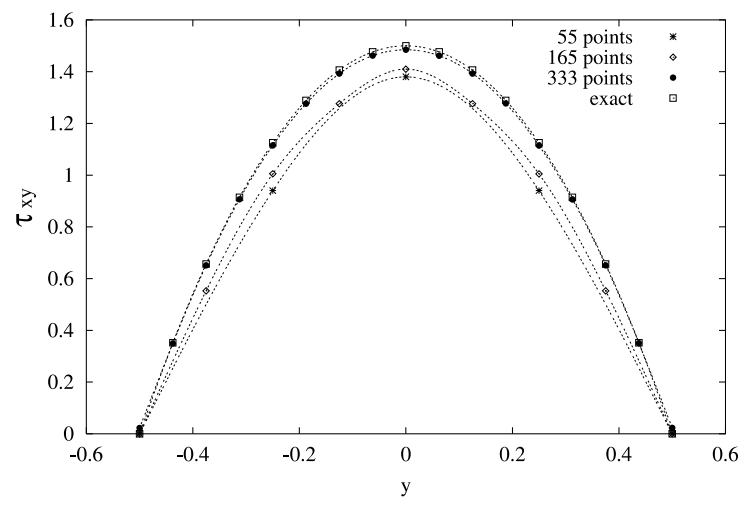

Fig. 12. Cantilever beam. Convergence of the shear stress distribution at $x=L / 2$ for different regular grids.
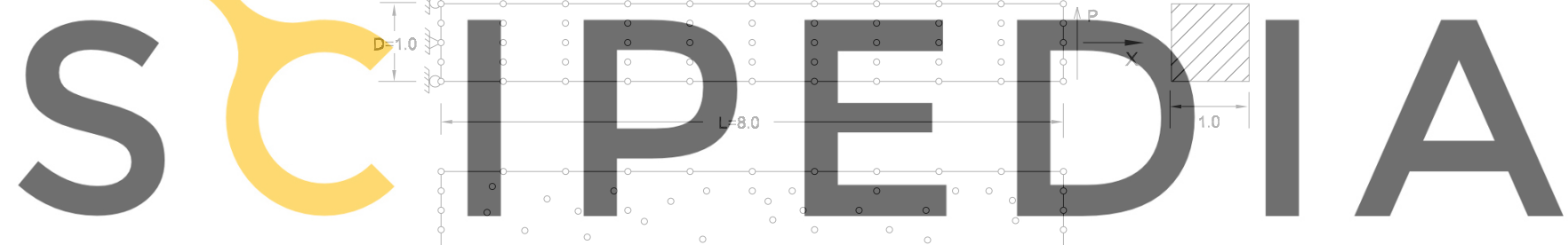

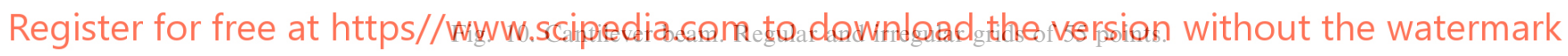

(a)

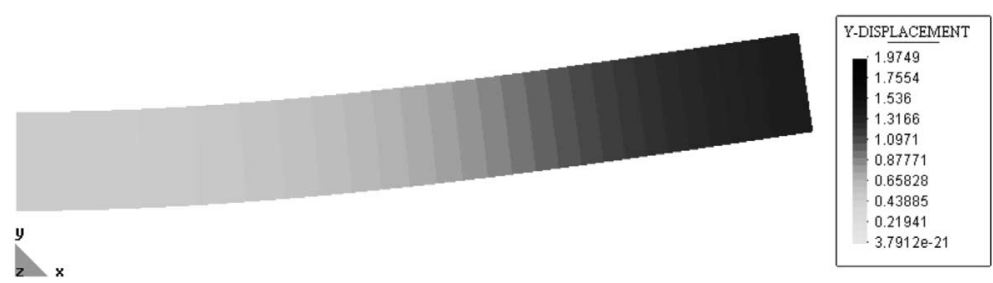

(b)

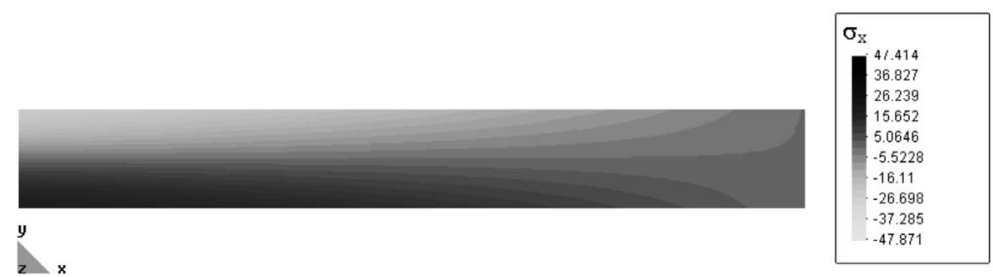

Fig. 11. Cantilever beam analyzed with a regular grid of 333 points. (a) Contours of the vertical displacement; (b) contours of the $\sigma_{x}$ stress. 


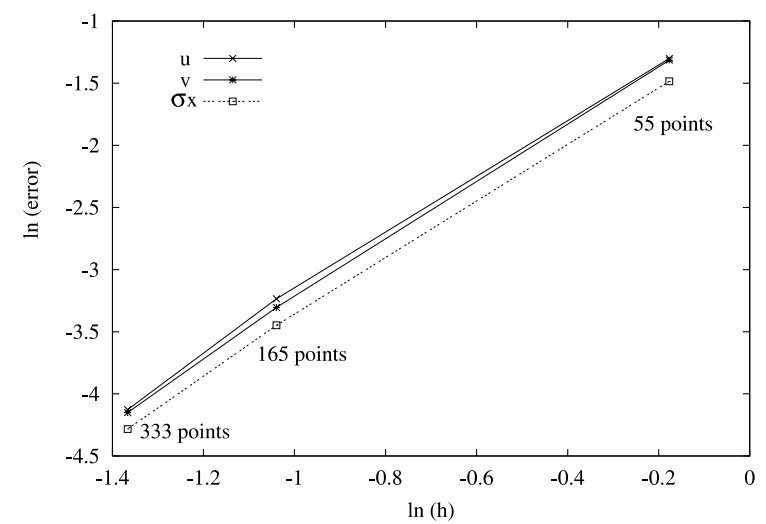

Fig. 13. Cantilever beam with regular point distribution. Convergence of displacements and $\sigma_{x}$ stress with the average radial distance for all clouds.

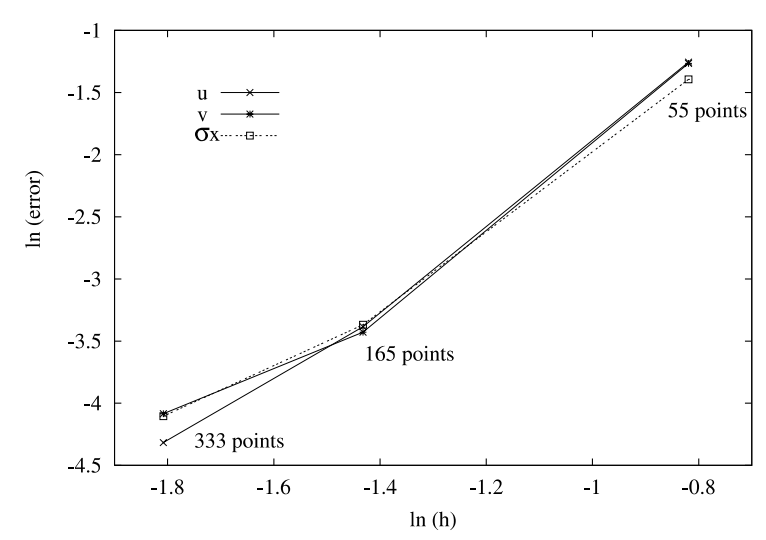

Fig. 14. Cantilever beam with irregular distribution of points. Convergence of displacements and $\sigma_{x}$ stress with the average radial distance for all clouds.

A comparison of relevant displacement and stress values with the analytical solution obtained from Ref. [28] is presented in Table 5.

(a)

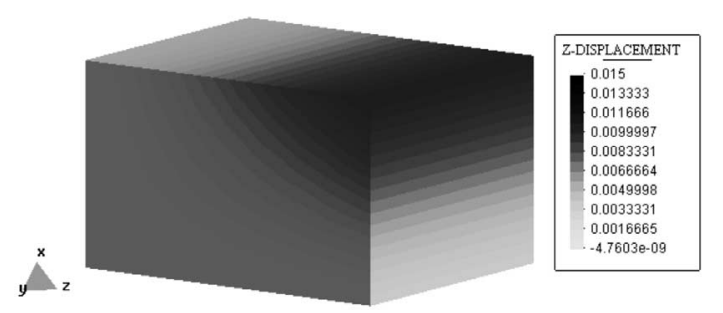

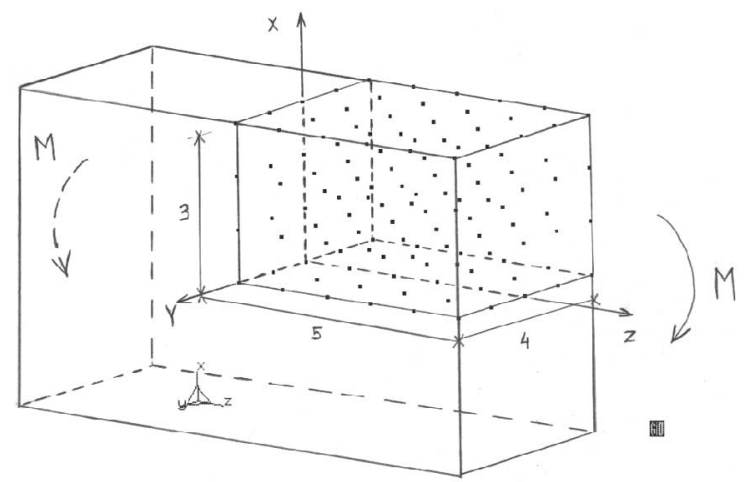

Fig. 15. Prismatic solid under end bending moment. Geometry, loading and arrangement of points $E=1000, v=0.25$.

Finally, Fig. 17 shows the contours of the displacement along the $z$-axis and the $\sigma_{z}$ stress obtained without the stabilization terms. Note that the solution drastically deteriorates in this case, as expected.

\section{Conclusions}

The stabilized FPM using the FIC procedure is a promising numerical method for the meshless solution of problems in solid mechanics. The stabilization terms were found to be crucial to obtain a smooth solution in all cases studied. Results for the 2D and 3D elasticity problems analyzed with the stabilized quadratic FPM yielded a higher accuracy than those obtained with standard FEM. The accuracy was remarkably higher for the stress values. The optimal selection of the stabilization parameters and the validation of the stabilized FPM for problems involving heterogeneous materials and complex $3 \mathrm{D}$ geometries are the main challenges in the extension and validation of the new meshless procedure.

(b)

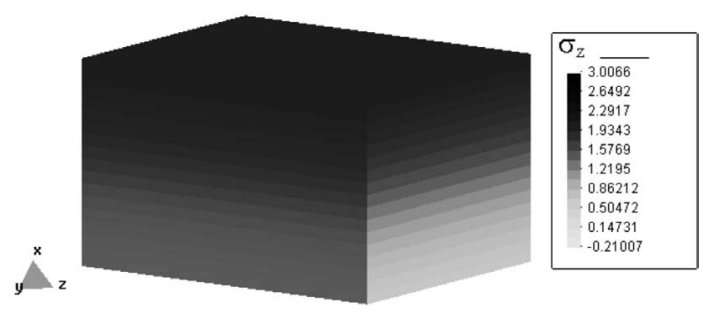

Fig. 16. Prismatic solid under end bending moment analyzed with the stabilized FPM. (a) Contours of $z$ displacement; (b) contours of $\sigma_{z}$ stress. 
Table 5

Numerical and analytical results for prismatic solid under end bending moment ${ }^{\mathrm{a}}$

\begin{tabular}{llll}
\hline & $u$ & $w$ & $\sigma_{z}$ \\
\hline FPM 120 points (regular grid) & -0.0138 & 0.015 & 3.0066 \\
FPM 271 points (irregular grid) & -0.0139 & 0.014991 & 3.0086 \\
Analytical [28] & -0.013625 & 0.015 & 3.0 \\
\hline
\end{tabular}

${ }^{\text {a }}$ Figures show maximum displacement and maximum stress values.

(a)

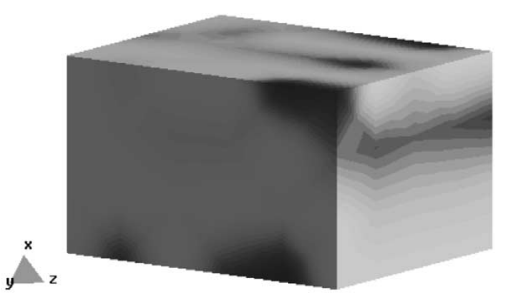

(b)

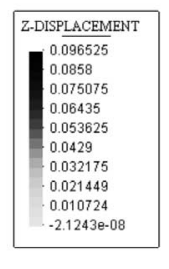

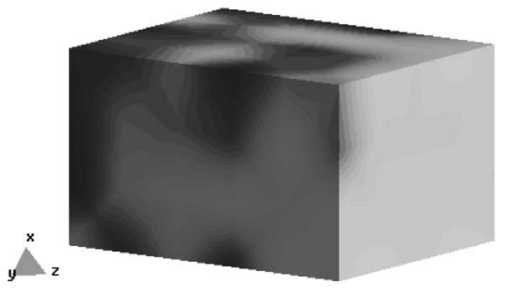

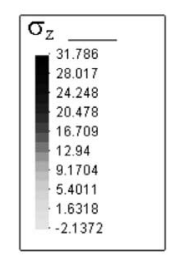

Fig. 17. Prismatic solid under end bending moment analyzed with the FPM without the stabilization procedure. (a) Contours of $z$ displacement; (b) contours of $\sigma_{z}$ stress.

\section{Acknowledgements}

The authors are thankful to Mr. Carlos Sacco and Prof. Sergio Idelsohn for many useful discussions.

\section{References}

[1] Monaghan JJ. Smoothed particle hydrodynamics. Annu Rev Astron Phys 1992;30:543-74.

[2] Randles PW, Libersky LD. Smoothed particle hydrodynamics: some recent improvement and applications. Appl Mech Engng 1996;139:1-4.

[3] Perrone N, Kao R. A general finite difference method for arbitrary meshes. Comput Struct 1975;5:45-7.

[4] Liszka T, Orkisz J. The finite difference method at arbitrary irregular grids and its application in applied mechanics. Comput Struct 1980;11:83-95.

[5] Nayroles B, Touzot G, Villon P. Generalizing the FEM: diffuse approximation and diffuse elements. Comput Mech 1992;10:307-18.

[6] Belytschko T, $\mathrm{Lu} \mathrm{Y,} \mathrm{Gu} \mathrm{L.} \mathrm{Element} \mathrm{free} \mathrm{Galerkin}$ methods. Int J Numer Meth Engng 1994;37:229-56.

[7] Dolbow J, Belytschko T. An introduction to programming the meshless element free Galerkin method. Arch Comput Meth Engng 1998;5(3):207-41.

[8] Liu WK, Chen Y, Jun S, Chen JS, Belytschko T, Pan C, Uras RA, Chang CT. Overview and applications of the Reproducing Kernel particle method. Arch Comput Meth Engng 1996;3(1):3-80.

[9] Atluri SN, Zhu T. A new meshless local Petrov-Galerkin (MLPG) approach in computational mechanics. Comput Mech 1998;22:117-27.

[10] Atluri SN, Kim H, Cho JY. A critical assessment of the truly meshless local Petrov-Galerkin (MLPG) and local boundary integral equation (LBIE) methods. Comput Mech 1999;24(5):348-73.

[11] De S, Bathe KJ. The method of finite spheres. Comput Mech 2000;25:329-45.

[12] Oñate E, Idelsohn S, Zienkiewicz OC, Fisher T. A finite point method for analysis of fluid flow problems. Proc Ninth Int Conf Finite Element Methods in Fluids, Venize, Italy, 15-21 October, 1995.

[13] Oñate E, Idelsohn S, Zienkiewicz OC, Taylor RL. A finite point method in computational mechanics. Applications to convective transport and fluid flow. Int $\mathbf{J}$ Numer Meth Engng 1996;39:3839-66.

[14] Oñate E, Idelsohn S, Zienkiewicz OC, Taylor RL. A stabilized finite point method for analysis of fluid mechanics problems. Comput Meth Appl Engng 1996;139(1-4): 315-47.

[15] Oñate E, Idelsohn S. A mesh free finite point method for advective-diffusive transport and fluid flow problems. Comput Mech 1988;21:283-92.

[16] Oñate E, Sacco C, Idelsohn S. A finite point method for incompressible flow problems. Comput Visual Sci 2000; 3:67-75.

[17] Taylor RL, Idelsohn S, Zienkiewicz OC, Oñate E. Moving least square approximations for solution of differential equations. Research Report 74, CIMNE, Barcelona, 1995.

[18] Oñate E. Derivation of stabilized equations for advectivediffusive transport and fluid flow problems. Comput Meth Appl Mech Engng 1998;151(1-2):233-67.

[19] Oñate E, García J, Idelsohn S. Computation of the stabilization parameter for the finite element solution of advective-diffusive problems. Int J Numer Meth Fluids 1997;25:1385-1407.

[20] Oñate E, García J, Idelsohn S. In: Ladeveze P, Oden JT, editors. An alpha-adaptive approach for stabilized finite element solution of advective-diffusive problems with sharp 
gradients. New Advances in Adaptive Comp Meth Mech, Amsterdam: Elsevier; 1998.

[21] Oñate E, Manzan M. A general procedure for deriving stabilized space-time finite element method for advectivediffusive problems. Int J Numer Meth Fluids 1999;31:20321.

[22] Oñate E. A finite element method for incompressible viscous flows using a finite increment calculus formulation. Comput Meth Appl Mech Engng 2000;182:355-70.

[23] Oñate E, García J. A stabilized finite element method for incompressible viscous flows with surface waves using a finite calculus procedure. Comput Meth Appl Mech Engng, in press.

[24] Oñate E, Perazzo F, Miquel J. In: W. Wunderlich et al., editor. Advances in the stabilized finite point method for structural mechanics. Publication No. 164, CIMNE, Barcelona, May 1999; European Conference on Computational Mechanics (ECCM'99), 31 August-3 September, 1999, Munich, Germany.

[25] Zienkiewicz OC, Taylor RL. The finite element method. 5th ed. Three volumes. London: Butterworth-Heinemann; 2000.

[26] Beissel S, Belytschko T. Nodal integration of the elementfree Galerkin method. Comput Meth Appl Mech Engng 1996;139:49-74.

[27] Bonet J, Kulasegarm S. Finite increment gradient stabilization of point integrated meshless method for elliptic equations. Commun Numer Meth Engng 2000;16:475-83.

[28] Timoshenko SP, Goodier JN. Theory of elasticity. 3rd ed. New York: Mc-Graw Hill; 1970. 